\title{
A numerical study of dynamic tunability of perfect absorption with temperature in the visible region based on a nanostructure containing multilayer graphene ${ }^{\text {th }}$
}

\author{
Xiuli Jia ${ }^{\mathrm{a}}$, Xiaoou Wanga,*, Qingxin Meng ${ }^{\mathrm{a}}$, Chengxun Yuan ${ }^{\mathrm{a}}$, Zhongxiang \\ Zhou $^{\mathrm{a}}$ \\ ${ }^{a}$ Harbin institute of technology, Harbin 150001, China
}

\begin{abstract}
We present a multilayer graphene-embedded nano-cross elliptical hole (MGENCEH) structure that has dynamic tunability of perfect absorption (PA) with temperature in the visible region. The results of the numerical simulation show that the structure can realize absorption that retains super-99.9\% over a wide incident angle of both transverse electric (TE) and transverse magnetic ( $\mathrm{T}$ M) polarization light. This PA is higher than the results of Zhou et al. [doi: 10.1364/OE.23.00A413]. The simulation shows that the absorption bands of PA can be broadened more than six times larger than no graphene structure. The dynamic tunability of PA with temperature in the visible region is not a completely linear relationship as compared to the terahertz region [doi: 10.1140/epjb/e2015-60233-0]. This mechanism of PA is mainly caused by hybrid plasmon absorption at higher temperatures and exciton absorption at lower temperatures. The simple and flexible PA is particularly desirable for various potential applications such as solar energy absorbers.

Keywords: Nanostructure, Perfect absorption, Graphene, Hybrid plasmon absorption, Exciton absorption.

2015 MSC: 00-01, 99-00
\end{abstract}

\footnotetext{
National Natural Science Foundation of China (No 61205011), National Science Foundation of China (NSFC) (No. 61205093), Fundamental Research Funds for the Central Universities (Grant No. HIT. MKSTISP. 2016 11).

* Corresponding author

Email address: wxo@hit.edu.cn (Xiaoou Wang)
}

Preprint submitted to Journal of LATEX Templates

April 7, 2016 


\section{Introduction}

Extensive effort on perfect absorption (PA) has been expended in the development of various plasmon structures such as nanodisks, nanowires, nanocones, and nanohole arrays. These structures have displayed unique optical and elec-

5 trical characteristics for energy harvesting, but there are no dynamic tunable

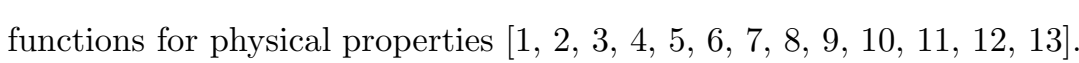

Recent studies have shown that graphene-embedded plasmon structures have strong dynamic tunable functions. Graphene is a two-dimensional, one-atomthick material with electrically tunable conductivity and a high carrier mobility

10 in the terahertz [14] and near-infrared regions [15]. These features promote the application of graphene in compact, dynamic, tunable modulators. The interaction between graphene and optical waves is rather weak, but plasmon structures have demonstrated exotic performance in boosting light-matter interactions.

By embedding graphene in plasmon structures, it is possible to enhance the

15 interaction between plasmon structures and incident electromagnetic waves, so that we can obtain tunable metamaterials [16, 17, 18], absorbers [19, 20, 21], and sensors [22]. Layered graphene structures have different functions depending on the number of layers, such as tunneling transistors using monolayer graphene [23] or optical modulators [24], which have been realized experimentally by

20 double-layer structures, where massless Dirac fermions of two separate layers are coupled only through many-body Coulomb interactions, tunable infrared plasmonic devices [25], and hyperbolic metamaterials [26] by multilayer graphene structures. Special attention has been paid to double-layer systems which considering their plasmon modes for doped [27, 28]. The enhancement effect of 25 the interaction among different charge carriers was found, that is, the tunneling between graphene and two-dimensional electron gas plays a significant role in determining the number of plasmon modes, as well as their dispersions under certain parameter regimes. Coexistence such as in Dirac/Schrodinger hybrid electron systems have been directly experimentally observed [29]. It has been 
shown that the surface plasmons in graphene can be significantly influenced by many particle effects involving interactions between electrons and plasmons. A deep understanding of the coupling between charge carriers and plasmons in graphene is highly desirable because of the significant potential of graphene in plasmonics [30]. Coupling between plasmons in graphene and electron gas in

35 metal-like substrates have been investigated by both numerical calculation [31] and theoretical analysis [32, 33].

The visible region accounts for more than $40 \%$ of sunlight, and thus, the development of optical absorption in the visible region is very important. In this paper, we propose a perfect absorber (PA) in the visible region based on a

40 multilayer graphene-embedded nano-cross elliptical hole (MGENCEH) structure in part 2. The complex physical properties of graphene at different temperatures are calculated in part 3. We simulate this MGENCEH structure by the threedimensional (3D) finite difference time domain (FDTD) method and obtain the absorption, transmission, and reflection of $0^{\circ}, 30^{\circ}$, and $60^{\circ}$ incidence for both

45 transverse electric (TE) wave and transverse magnetic (TM) wave polarization light. The dynamic tunable function of graphene with different temperatures is numerically calculated in parts 4 and 5 , and the physical mechanism is analyzed in part 6.

\section{Structure design}

We present the dynamic tunability of PA by temperature in the visible region based on an MGENCEH structure, as shown in Fig. 1. A similar structure was fabricated using standard lift-off electron-beam lithography [34, 35, 36]. The basic structure of MGENCEH is a five-layer structure of metal $(\mathrm{Au}) / \operatorname{dielectric}\left(\mathrm{SiO}_{2}\right) /$ $\operatorname{metal}(\mathrm{Au}) / \operatorname{dielectric}\left(\mathrm{SiO}_{2}\right) / \operatorname{metal}(\mathrm{Au})(\mathrm{MDMDM})$. Layer 5 on the bottom is a metal functional layer, layer 4 is a dielectric layer, layer 3 is the elliptical nanohole metal layer with an angular offset of $\alpha=22.5^{\circ}$ from the $x$-axis, layer 2 is another dielectric layer between layers 3 and 1, and layer 1 has an elliptical nanohole with an angular offset of $\beta=45^{\circ}$ from the major of elliptical nanohole 
of layer 3. The semi-major $\left(r_{1}\right)$ and semi-minor $\left(r_{2}\right)$ axes of the elliptical holes are 150 and $115 \mathrm{~nm}$, respectively. These geometric structure parameters are from Kang et al. [35]. The graphene layers are properly embedded in different positions (2 to 5). TE-polarization light or TM-polarization light is incident to this nanostructure from $0^{\circ}$ to $60^{\circ}$.

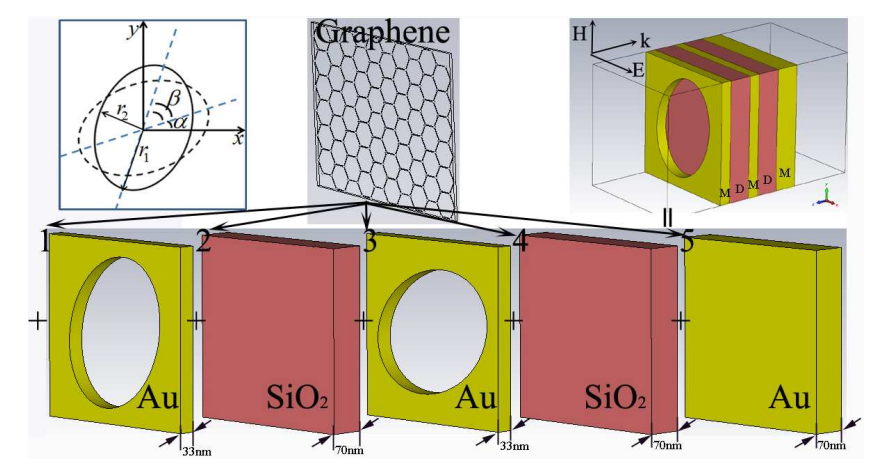

Fig. 1. Schematic of two metal films perforated with identical elliptical holes with angular offsets of $\alpha=22.5^{\circ}$ and $\beta=45^{\circ}$. The two metal films are separated by a dielectric layer. The dashed and solid ellipses indicate the lower and upper elliptical holes, respectively, with semi-major $\left(r_{1}\right)$ and semiminor $\left(r_{2}\right)$ axes of 150 and $115 \mathrm{~nm}$, respectively. The unit cell is arranged in a square lattice with a lattice constant of $350 \mathrm{~nm}$. Graphene layers are embedded in positions 2 to 5 .

Herein, Au (optical) is chosen as the metal material, and the dielectric material chosen is $\mathrm{SiO}_{2}$ (optical) with relatively low permittivity, which is an ideal candidate for achieving high absorption [37, 38]. The thickness of the bottom functional layer is larger than the penetration depth of optical waves in the visible region, and thus this functional layer will block any incident light transmitted through the layers before the functional layer. The functional layer

70 leads to zero transmission in the working range; therefore, we can obtain PA. Graphene is considered a sheet material with complex surface conductivity $(\sigma)$ because a one-atom-thick graphene sheet is sufficiently thin as compared to the 
considered wavelength. The complex conductivity of graphene can be described by the Drude model [39]. In the simulation, we used the 3D finite difference

75 time domain (FDTD) method (CST Studio Suite 2013). The periodic boundary conditions are used in both the $\mathrm{X}$ and $\mathrm{Y}$ directions, and a perfect matching layer boundary condition is used in the $\mathrm{Z}$ direction.

\section{Graphene's complex physical properties at different temperatures}

The conductivity $(\sigma)$ of graphene comprises the contributions of both interso band $\left(\sigma_{\text {inter }}\right)$ and intraband $\left(\sigma_{\text {intra }}\right)$ transitions [38].

$$
\begin{gathered}
\sigma_{\text {inter }}\left(\omega, \mu_{c}, \Gamma, 0\right)=-\frac{i e^{2}}{4 \pi \hbar} \ln \left(\frac{2\left|\mu_{c}-(\omega-i 2 \Gamma) \hbar\right|}{2\left|\mu_{c}+(\omega-i 2 \Gamma) \hbar\right|}\right), \\
\sigma_{\text {intra }}\left(\omega, \mu_{c}, \Gamma, T\right)=-\frac{i e^{2} k_{B} T}{\pi \hbar^{2}(\omega-i 2 \Gamma)}\left[\frac{\mu_{c}}{k_{B} T}+2 \ln \left(e^{-\mu_{c} / k_{B} T}+1\right)\right] .
\end{gathered}
$$

Here, $e, k_{B}$, and $\hbar$ are the electron charge, Boltzmann constant, and reduced Planck constant, respectively, and $u_{c}$ and $T$ are the chemical potential and temperature of graphene, respectively. $\Gamma$ is the electron-phonon scattering rate,

85 which is $0.43 \mathrm{meV}$ (refer to the parameter settings in Ref. [40]).

$$
\sigma=\sigma_{\text {inter }}+\sigma_{\text {intra }}
$$

Then, we can obtain the complex permittivity of graphene according to the functional relationship

$$
\varepsilon=1+i \sigma /\left(\varepsilon_{0} \omega t\right)
$$

where $\varepsilon_{0}$ is the vacuum permittivity and $t$ is the thickness of the graphene layer, which is assumed to be $1 \mathrm{~nm}$ in this paper.

$$
\tan \delta=\operatorname{imag}(\varepsilon) / \operatorname{real}(\varepsilon)
$$




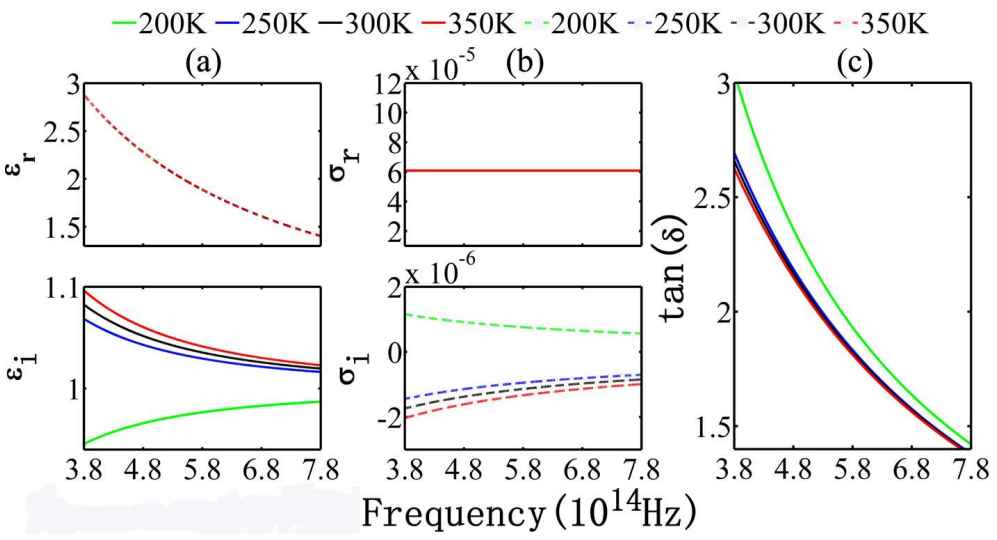

Fig. 2. Graphene's complex physical properties at $T=200,250,300$, and 350 $\mathrm{K}, u_{c}=5 \mathrm{meV}$, and $f$ from 3.8 to $7.8 \times 10^{14} \mathrm{~Hz}$ : (a) Permittivity (real and imagined part of $\varepsilon$ ), (b) conductivity (real and imagined part of $\sigma$ ), and (c) loss tangent $(\tan \delta)$.

In our calculations, we assumed a fixed chemical potential $\left(u_{c}=5 \mathrm{meV}\right)$. Under such an assumption, we calculated graphene's complex permittivities $(\varepsilon)$, conductivities $(\sigma)$, and loss tangents $(\tan \delta)$. These physical properties are tuned by $T$, as shown in Fig. 2. In the visible region (3.8 to $7.8 \times 10^{14}$ $\mathrm{Hz})$, as shown in Fig. 2(a), the real parts of permittivity $\left(\varepsilon_{r}\right)$ decrease with 95 increasing frequency and the imagined parts of permittivity $\left(\varepsilon_{i}, T=350,300\right.$, and $250 \mathrm{~K}$ ) also decrease with increasing frequency or decreasing temperature except at $T=200 \mathrm{~K}$. The $\varepsilon_{i}$ value at $200 \mathrm{~K}$ has a symmetric relation with the permittivity of the other temperatures under $\varepsilon_{i}=1$. As shown in Fig. 2(b), the real parts of conductivity $\left(\sigma_{r}\right)$ exhibit no change with increasing temperature or frequency, that shows small ohmic losses in visible light. The imagined parts of conductivity $\left(\sigma_{i}, T=350,300\right.$, and $\left.250 \mathrm{~K}\right)$ increase with increasing frequency or decreasing temperature except at $T=200 \mathrm{~K} . \sigma_{i}$ has a symmetric relation with the conductivity of other temperatures under $\sigma_{i}=0$. Moreover, we notice that graphene's loss tangent $(\tan \delta)$ has a little sensitivity to the temperature, but when the temperature decreases to $200 \mathrm{~K}$, $\tan \delta$ exhibits significant change, as shown in Fig. 2(c). As compared to traditional metals, graphene has dynamic tunable physical properties that depend on temperature, as shown in Fig. 2. 
This makes it a promising candidate for the design of tunable metamaterials and device applications [41].

4. Dynamic tunable PA spectrum over a wide incident angle of TEpolarized light

Using the rigorous electromagnetic analysis method, i.e., the 3D finite difference time domain (FDTD) algorithm, we observe that the absorption spectrum shows excellent sensitivity to the incident polarization state (TE and TM). The

115 absorption $(A=1-R-T)$ spectrum of the periodic nano-cross elliptical hole structure under TE-polarized light excitation $\left(0^{\circ}, 30^{\circ}, 60^{\circ}\right.$ incident $)$ can be calculated. For $0^{\circ}$ and $30^{\circ}$ incident light, we can achieve PA over $99.9 \%$ in some of the visible region, but for $60^{\circ}$ incident, we do not find a band. Figs. 3 and 4 respectively show the different absorption spectra and the dynamical tunable

120 functions of graphene at different temperatures under TE-polarized excitation.

\section{1. $0^{\circ}$ incidence}

Under $0^{\circ}$ incidence of TE-polarized light in the MGENCEH structure, the dynamical tunable PA at temperatures of 350, 300, 250, and $200 \mathrm{~K}$ are shown in Fig. 3. 


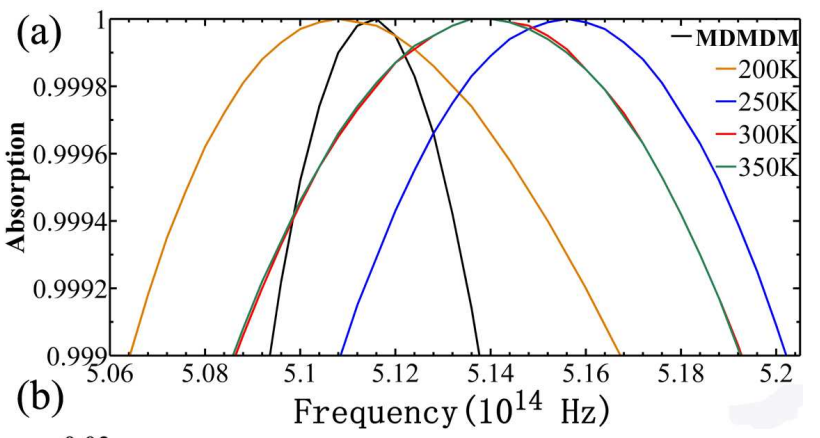

(b)

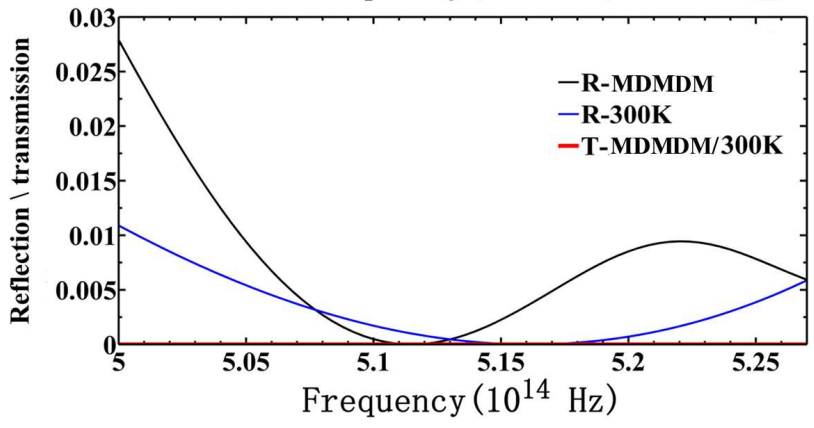

Fig. 3. Under $0^{\circ}$ incidence of TE-polarized light: (a) Absorption spectrum and (b) reflection and transmission spectra.

As shown in Fig. 3(a), the PA band of the MGENCEH structure for the $0^{\circ}$ incidence of TE-polarized light has a band that is 3.5 times broader that of MDMDM (from $5.06 \times 10^{14} \mathrm{~Hz}$ to $5.21 \times 10^{14} \mathrm{~Hz}$ at $T=200,250,300$, and $350 \mathrm{~K})$. For no graphene, i.e., the MDMDM structure, which has a narrow absorption frequency band from $5.0865 \times 10^{14} \mathrm{~Hz}$ to $5.1285 \times 10^{14} \mathrm{~Hz}$.

The PA band is blue-shifted as compared to the MDMDM structure at room temperature $(300 \mathrm{~K})$, and the band broadens greatly at the same time. The PA band at $350 \mathrm{~K}$ remains stable as compared to that at $300 \mathrm{~K}$. On the other hand, when we reduce the temperature to $250 \mathrm{~K}$, the PA band obviously red-shifts. When we continue to reduce the temperature to $200 \mathrm{~K}$, the PA band blue-shifts 135 again. The dynamic tunability of PA using temperature in the visible region is not a completely linear relationship as compared to the terahertz region [42].

As shown in Fig. 3(b), electromagnetic energy can be absorbed totally at resonance, whereas absorption decreases sharply as the frequency deviates from 
the resonant frequency. For example, as shown in Fig. 3(b), the reflection 140 and transmission are zero $(R=0, T=0)$, which means that the absorption is approximately $100 \%$ at the resonance frequency, and the reflection is enhanced outside the region of the resonance frequency.

\section{2. $30^{\circ}$ incidence}

As shown in Fig. 4(a), the PA band of the MGENCEH structure for the $30^{\circ}$ incidence of TE-polarized light is six times broader than that of the MDMDM structure. The MDMDM structure has a narrow PA frequency band from $6.165 \times 10^{14} \mathrm{~Hz}$ to $6.1955 \times 10^{14} \mathrm{~Hz}$, whereas the MGENCEH structure has a PA band from $6.05 \times 10^{14} \mathrm{~Hz}$ to $6.25 \times 10^{14} \mathrm{~Hz}$. We could see some details of tune function on absorption value by tuning the temperature of the MGENCEH structure under $30^{\circ}$ incidence of TE-polarized light. The absorption value of MGENCEH improved especially at $T=200 \mathrm{~K}$.
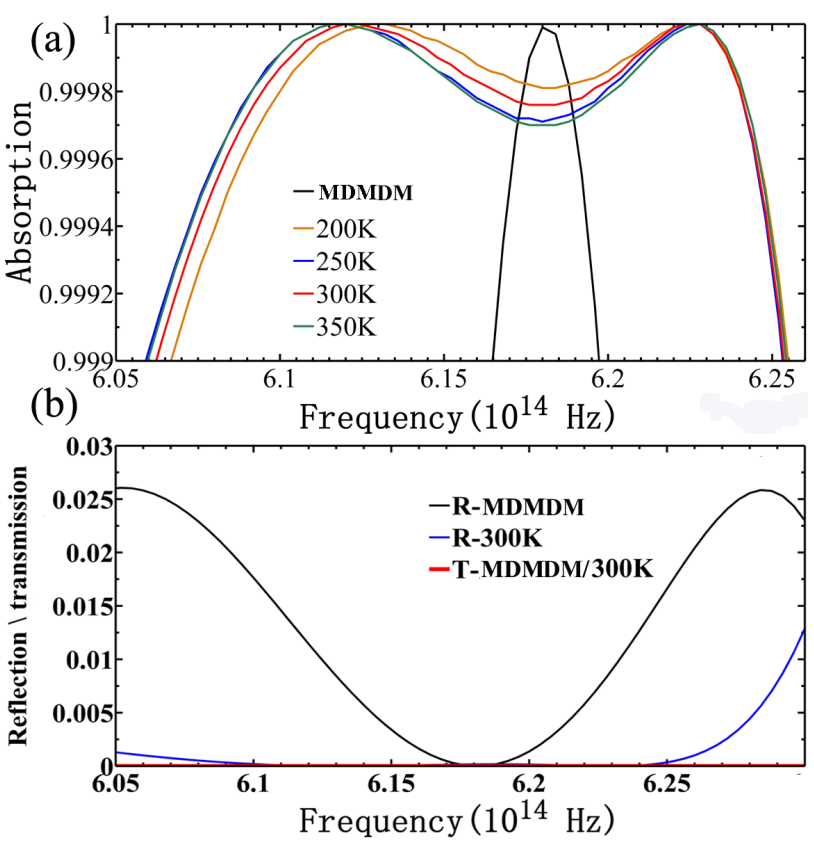
Fig. 4. Under $30^{\circ}$ incidence of TE-polarized light: (a) Absorption spectrum and (b) reflection and transmission spectra.

As shown in Fig. 4(b), by tuning the temperature of graphene at $300 \mathrm{~K}$, the reflection $(R)$ of the MGENCEH structure is zero from 6.1 to $6.25 \times 10^{14}$ $\mathrm{Hz}$, and this frequency region has many times compared with the region of zero reflection of MDMDM structure. According to the relationship of $A=1-R-T$, this broad zero-reflection band means that the MGENCEH structure improved the width of the PA band.

\section{Dynamic tunable PA spectrum over a wide incident angle of the TM-polarized light}

Under $0^{\circ}, 30^{\circ}$, and $60^{\circ}$ incidence of TM-polarized light excitation, we also achieved PA over $99.9 \%$ in some of the visible regions. Figs. 5-7 show that the different absorption spectra and dynamical tunable functions depend on the temperature of graphene under TM-polarized excitation.

\section{1. $0^{\circ}$ incidence}

As shown in Fig. 5(a), the PA bands of the MGENCEH structure with different temperatures under $0^{\circ}$ incidence of TM-polarized light are more than six times broader than those of the MDMDM structure. The MDMDM structure has two narrow absorption frequency bands at approximately $5.95 \times 10^{14} \mathrm{~Hz}$ and $6.16 \times 10^{14} \mathrm{~Hz}$. The MGENCEH structure continuously makes two narrow PA

170 bands. The PA band greatly broadens from $5.9 \times 10^{14} \mathrm{~Hz}$ to $6.15 \times 10^{14} \mathrm{~Hz}$ when $T=200 \mathrm{~K}$, in contrast with the MDMDM structure.

When two metal particles are coupled, the dipoles of the MDMDM structure are hybridized and the electromagnetic interaction cannot be ignored, so the dispersion relation of the MDMDM structure will change significantly. The 175 surface plasmon resonance frequency split into symmetric mode (low-frequency mode) and asymmetric mode (high-frequency mode) [43], and so the absorption spectrum of the MDMDM structure performs multiple band absorption, which include the left PA band and right PA band for the $0^{\circ}$ incidence of TM-polarized 
light, as shown in Fig. 5(a). After we embed multilayer graphene into the MD180 MDM structure, the graphene inhibits the plasma frequency split and performs broad band absorption.

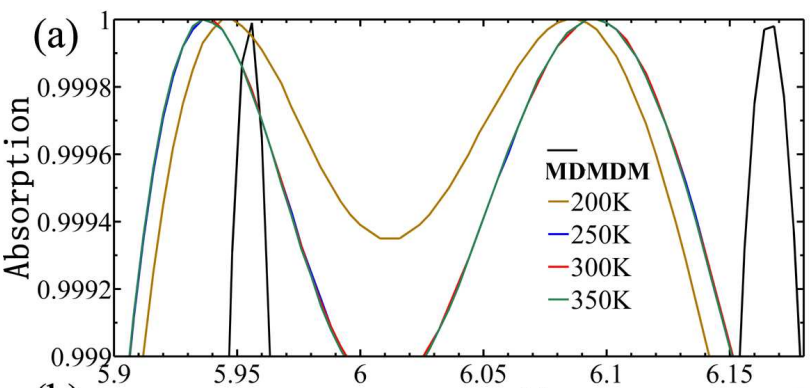

(b)

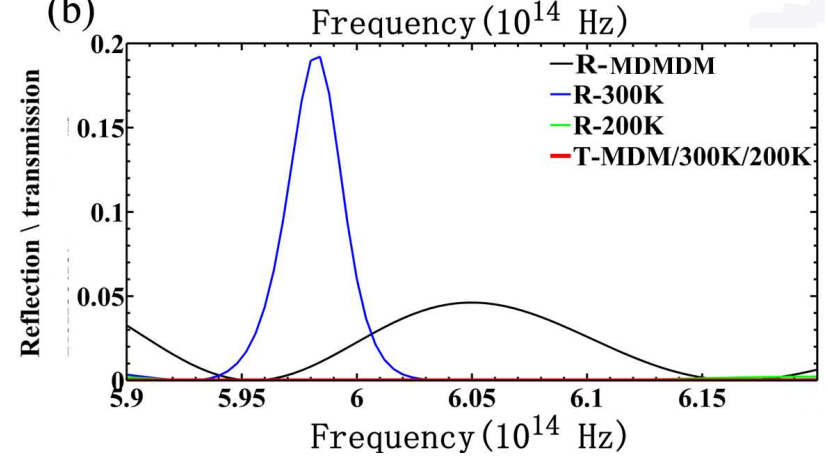

Fig. 5. Under $0^{\circ}$ incidence of TM-polarized light: (a) Absorption spectrum and (b) reflection and transmission spectra.

As shown in Fig. 5(b), the reflection of the MGENCEH structure is zero at frequencies other than $5.97 \times 10^{14} \mathrm{~Hz}$, which still has a reflection peak below 0.2. The absorption is still two broader absorption bands, in addition to that at the temperature of $200 \mathrm{~K}$. The reflection is very small, and this small reflection means that the MGENCEH structure can form a super-broad PA band, as shown in Fig. 5(a).

\section{2. $30^{\circ}$ incidence}

As shown in Fig. 6(a), the absorption of the MDMDM structure for the ${ }_{190} 30^{\circ}$ incidence of TM-polarized light has three narrow absorption bands, and the 

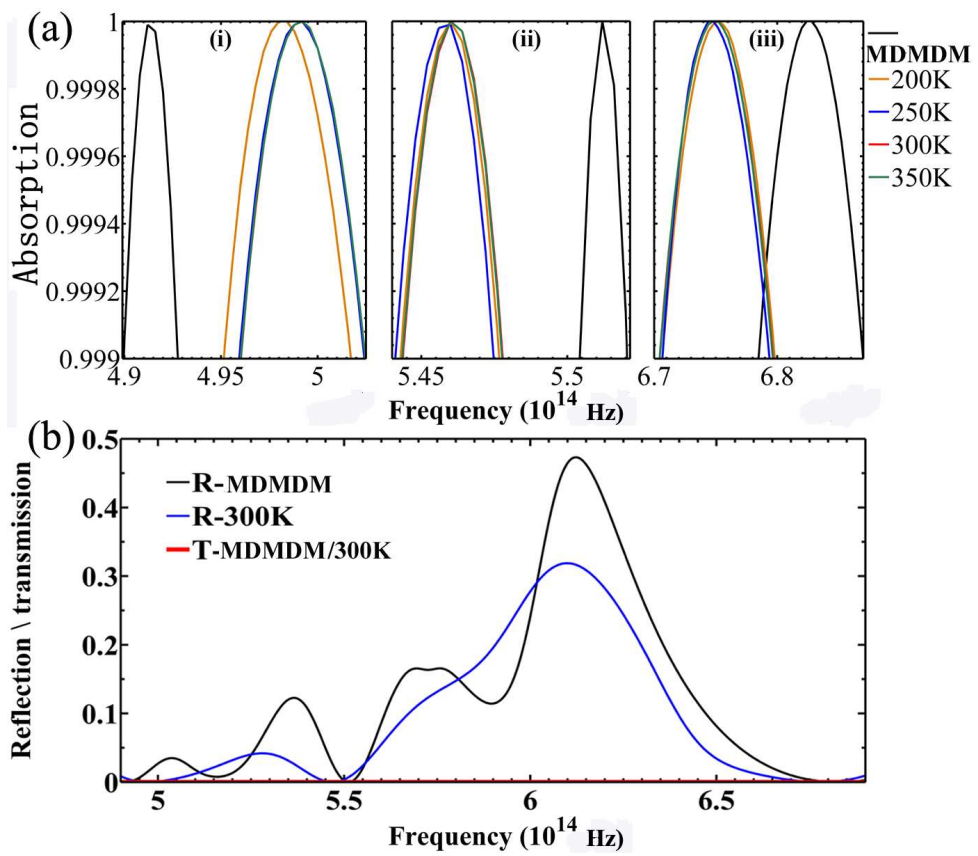

MGENCEH structure under $30^{\circ}$ incidence of TM-polarized light also has three absorption bands. The PA bands broadened 1.5 to 2 times when $T=200,250$, 300 , and $350 \mathrm{~K}$, in contrast with the MDMDM structure. Also, the absorption bands obviously blue-shift at lower-frequency regions (i) and red-shift at higher195 frequency regions (ii) and (iii), as shown in Fig. 6(a).

Fig. 6. Under $30^{\circ}$ incidence of TM-polarized light: (a) Absorption spectrum and (b) reflection and transmission spectra.

With the increase of incident angle, the absorption of the MDMDM structure is reduced, whereas the reflection increases. However, by embedding graphene, as show in Fig. 6(b), the reduction of the reflection is less obvious, which shows the good tunability of graphene on the absorption of the MGENCEH structure. 


\section{3. $60^{\circ}$ incident}

As shown in Fig. 7(a), the absorption for $60^{\circ}$ incidence of TM-polarized light has five very narrow absorption bands compared with no PA bands for $60^{\circ}$ incidence of TE-polarized light. For the MDMDM structure, the PA bands are very narrow and almost at the frequency points. The absorption bands of MGENCEH broaden 3 to 4 times when $T=200,250,300$, and $350 \mathrm{~K}$, in contrast to the MDMDM structure. Especially at $T=250 \mathrm{~K}$, the MGENCEH structure still has a tunable function at higher frequencies (iii), as shown in Fig. $7(\mathrm{a})$.
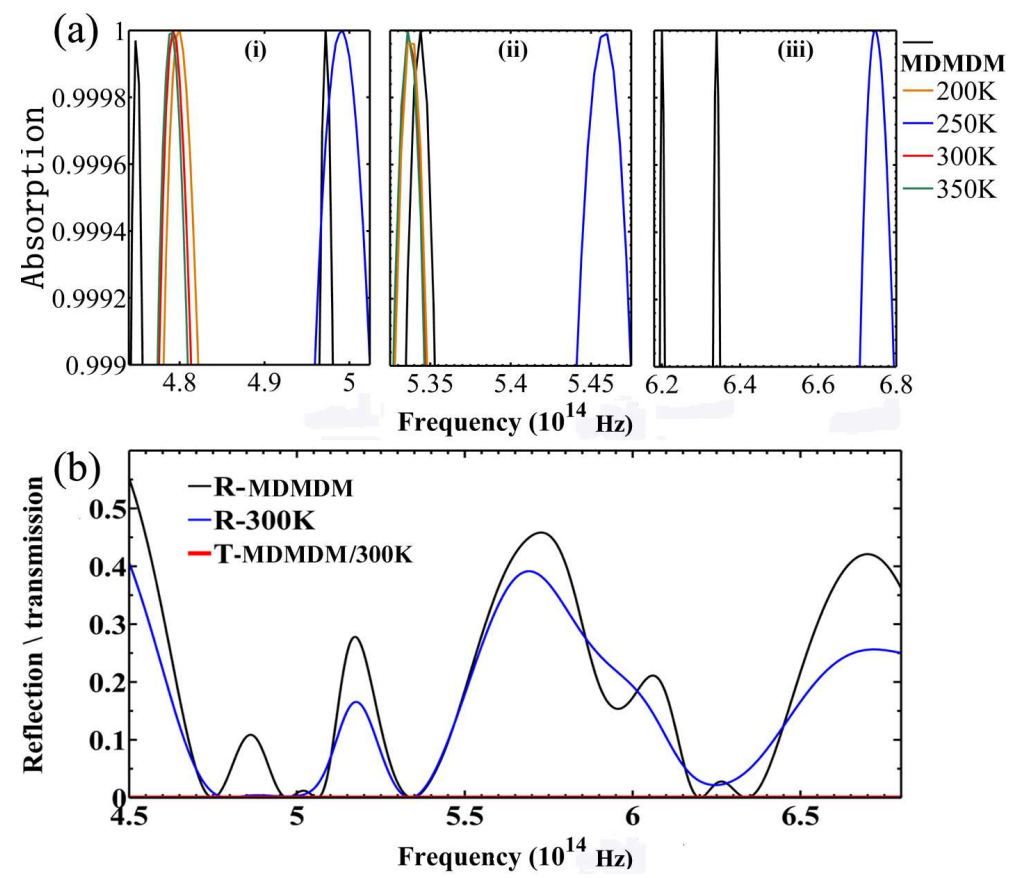

Fig. 7. Under $60^{\circ}$ incidence of TM-polarized light: (a) Absorption spectrum and (b) reflection and transmission spectra.

Continuing to increase the incident angle, as shown in Fig. 7(b), $R=300$ ${ }_{210} \mathrm{~K}$ also shows the good tunability of graphene for reducing the reflection as compared to the structure that does not contain graphene, i.e., the MDMDM 
structure.

\section{The physic mechanism analysis PA}

Parts 4 and 5 mainly introduced the temperature dependence of PA within a 15 relatively narrow band, but across the whole visible light range, the MGENCEH structure also shows broad-band nearly perfect absorption (BNPA), as shown in Figs. 8(a) and (b). Under the general temperature, the PA of the MGENCEH structure mainly depends on plasmon absorption, which is analyzed in part 6.1. However, at the lower temperature of $200 \mathrm{~K}$, the PA of the MGENCEH structure is affected and enhanced significantly by the temperature, and thus this PA at $200 \mathrm{~K}$ mainly depends on exciton absorption, which is analyzed in part 6.2.

\subsection{The hybrid plasmon absorption at higher temperatures $(T=250$ to $350 \mathrm{~K})$}

As shown in Figs. 8(a) and (b), the tunability of temperature is not obvious 225 across the large frequency range $\left(3.8 \times 10^{14} \mathrm{THz}\right.$ to $\left.7.8 \times 10^{14} \mathrm{THz}\right)$. However, the MGENCEH structure has BNPA as compared to the MDMDM structure, especially for TE-polarization light. Generally, it is believed that the absorption is polarization-independent for optical waves incident to nanostructures because only electrical component (TE) interacts with optical waves [44]. However, in this paper, the absorption shows that the interaction is also strong for TM-polarization light, i.e., the absorption is polarization-dependent. This polarization dependency is related to the nano-elliptical hole cross structure, which can produce the hybrid plasmon. 


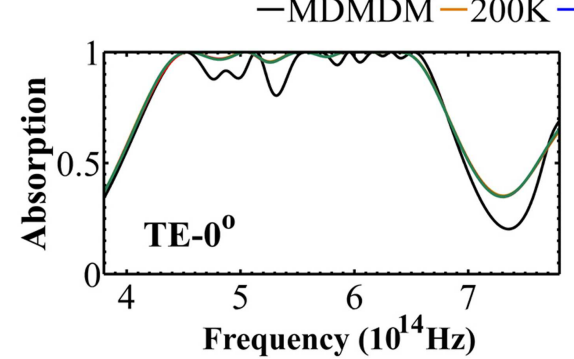

(a)

Electric Fild (A/m )

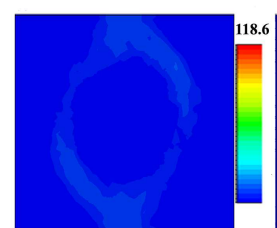

(i)

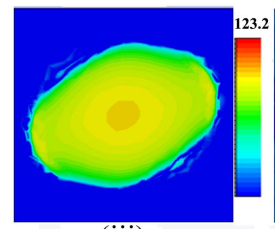

(iii)

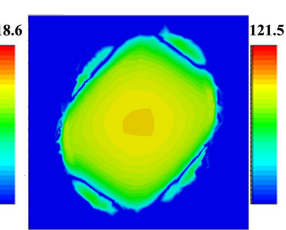

(ii)

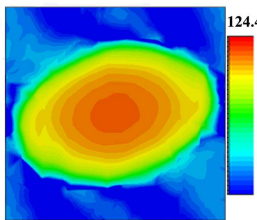

(iv)

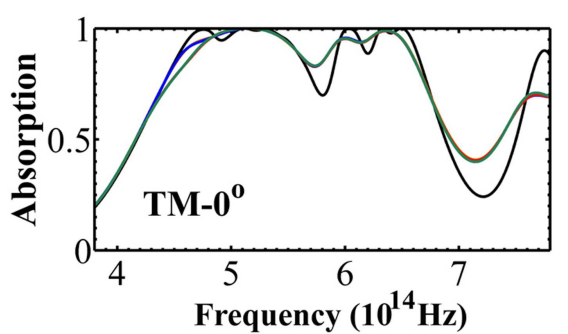

(b)

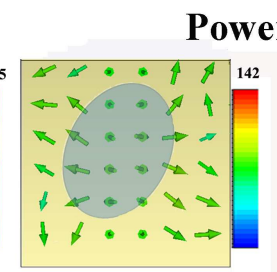

(i)

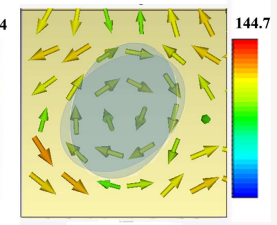

(iii)

(c)

(d)

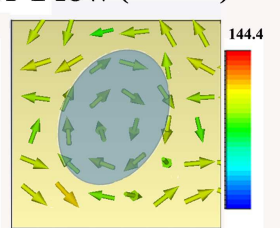

(ii)

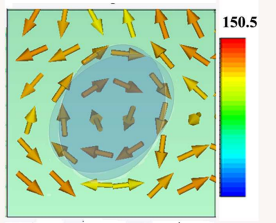

(iv)

Fig. 8. The hybrid plasmon absorption: (a) BNPA under $0^{\circ}$ of TE. (b) BNPA under $0^{\circ}$ of TM. (c) The electric field distribution of mono-elliptical structure (i), bis-elliptical structure (ii), MDMDM structure (iii) and MGENCEH structure (iv) for incident light. (d) The power flow of mono-elliptical structure (i), structure bis-elliptical (ii), MDMDM structure (iii), and MGENCEH structure (iv) for incident light.

In order to illustrate this physical insight of enhanced absorption mechanism 235 at higher temperatures (above $250 \mathrm{~K}$ ), we compared a series of models, as shown in Figs. 8(c) and (d). They are the mono-elliptical structure (i), bis-elliptical structure (ii), cross-elliptical structure (iii), i.e., the MDMDM structure, and multilayer graphene-embedded nano-cross elliptical hole structure (iv), i.e., the MGENCEH structure. 
As shown in Fig. 8(c), the maximum electric field distribution of (i) is 118.6 $\mathrm{A} / \mathrm{m}$; this value is enhanced in (ii), to $121.5 \mathrm{~A} / \mathrm{m}$, and enhanced again by (iii), to $123.2 \mathrm{~A} / \mathrm{m}$. It is finally enhanced by the MGENCEH structure (iv), whose maximum is $124.4 \mathrm{~A} / \mathrm{m}$.

The electric field of (i) shows that the plasmonic resonance is poorly excited 245 in the visible range. In contrast, on the $x-y$ plane, (ii), (iii), and (iv) induce the localized plasmonic resonance. Composite nanostructures are composed of two or more systems that have strong coupling effects between them due to their strong near-field electromagnetic interaction. Such composite structures therefore have richer plasmonic resonance properties, such as hybrid plasmon, than a single nanostructure (i), as shown in Fig. 8(c). Hybridized plasmon on a nanoscale concentrates the electromagnetic field energy and amplifies it locally. For incident light, the absorption of the MGENCEH structure (iv) around the resonant wavelength of $5.95 \times 10^{14} \mathrm{~Hz}$ is significantly enhanced up to $124.4 \mathrm{~A}$, and the absorption band is extended six times as compared to the absorption band of (iii), as shown in Fig. 5(a). This concentration and enhancement of the electromagnetic field by the MGENCEH structure is attributed to the hybridized plasmon, in which the local electric field is highly concentrated in the region of the elliptical hole, strengthening the light-graphene interaction significantly.

In addition to the local enhancement effect, the graphene also has the effect of reducing the optical dimensions. The structure of MGENCEH can control the optical dimensionsl from $3 \mathrm{D}$ to $2 \mathrm{D}$ by hybridized plasmon, so as to effectively control nanoscale optical transmission. As shown in Fig. 8(d), this hybridized plasmon leads to power flow from no circulation (i) to circulation (ii). The 265 maximum power flow intensity is from $142 \mathrm{VA} / \mathrm{m}^{2}$ to $144.4 \mathrm{VA} / \mathrm{m}^{2}$. It is then enhanced by (iii), whose maximum is $144.7 \mathrm{VA} / \mathrm{m}^{2}$, and then finally enhanced by the MGENCEH structure (iv), whose maximum is $150.5 \mathrm{VA} / \mathrm{m}^{2}$.

This enhancement by the MGENCEH structure results in PA and BNPA, as the mentioned above. On the one hand, this is because of the thickness of the film with increased, which inhibits the plasmon frequency split. On the other 
hand, it is because of the hybrid structure, which improves the interaction of the graphene and Au nanostructures with each other. The plasmon nanostructures can significantly enhance the local electric field strength of the interface, increase the interaction of graphene and light, and further improve the efficiency of light 275 absorption.

\subsection{The exciton absorption at lower temperatures $(T=200 \mathrm{~K})$}

In the visible range, the reflectivity per graphene layer is less than $0.1 \%$ and only about $2 \%$ per ten layers. The absorption rate increases $2.3 \%$ per layer of graphene. The graphene carrier mobility is hardly affected by temperature

280 about from $50 \mathrm{~K}$ to $500 \mathrm{~K}$. Thus, the absorption of the MGENCEH structure is mainly caused by the hybrid plasmon of the structure design and the enhanced plasmon effect of graphene. However, it was found that the absorption by some of semiconductor will cause absorption peaks at the low temperatures. When the incident photon energy is lower than the forbidden band width, the valence band

285 electrons are in the excited state but electrons and holes are bound together by the Coulomb force interaction. This bound state is known as excitons, which are electrically neutral and thus they cannot form a conduction current; however, they can move in the solid freely. The exciton absorption is generated when the incident photon energy is close to the valence band-exciton energy level spacing.

290 Generally, exciton absorption phenomena at low temperatures is relatively easily observed, and it is more obvious at lower temperature [45, 46, 47].

\section{Conclusion}

In conclusion, although traditional nanostructures such as the MDMDM structure can achieve PA, the PA band is narrow and non-tenable, which limits

295 the application of PA. Here, we present a MGENCH structure that can realize PA of dynamic tunable and broader bands. The absorption of the MGENCH structure is as high as super-99.9\%, and it sensitively depends on both TEand TM-polarized light in the visible region. The absorption is higher than the 
results of Zhou [4]. The resonant frequencies of the PA bands are dynamically tunable with changes to the temperature of the graphene layers; however, this tunability of PA is not a linear relationship as compared to the terahertz region [42]. For the wide incidence of TE- and TM-polarized light, the simulation results show that the bands of PA can be broadened more than six times with the increasing temperature of graphene as compared to the MDMDM structure. With increasing incidence angle, the width of the PA bands is reduced. The absorption for TE-polarized light better than that of TM-polarized light, but TM still has PA with $60^{\circ}$ as compared to TE-polarized light. The hybrid plasmon mechanism analysis of MGENCHS showed that a geometric design is a method of local field enhancement and that graphene can further enhance this local field. This PA is mainly caused by hybrid plasmons at higher temperatures and exciton absorption at temperatures lower than $200 \mathrm{~K}$. ACKNOWLEDGMENTS

This work was supported by the National Natural Science Foundation of China (No. 61205011), the National Science Foundation of China (NSFC) (No. 61205093) and the Fundamental Research Funds for the Central Universities (Grant No. HIT. MKSTISP. 2016 11).

\section{References}

[1] L. He, R. C. Jiang, D. Lai, H. Wang, Highly efficient si-nanorods/organic hybrid core-sheath heterojunction solar cells, Appl. Phys. Lett 99 (2011) 021104. doi:10.1063/1.3610461.

[2] O. Muskens, J. Rivas, R. Algra, E. Bakkers, A. Lagendijk, Design of light scattering in nanowire materials for photovoltaic applications, Nano Lett. 8 (2008) 2638-2642. doi:10.1021/n10808076.

[3] C. Lin, M. Povinelli, Optical absorption enhancement in silicon nanowire arrays with a large lattice constant for photovoltaic applications, Opt. Express 17 (2009) 19371-19381. doi:10.1364/OE.17.019371.

[4] H. Fang, X. Li, Y. X. S. Song, J. Zhu, Fabrication of slantingly-aligned 
silicon nanowire arrays for solar cell applications, Nat. Nanotechnol. 19 (2008) 255703. doi:10.1088/0957-4484/19/25/255703.

[5] M. Kelzenberg, D. Turner-Evans, B. Kayes, M. Filier, M. Putnam, N. Lewis, H. Atwater, Photovoltaic measurements in single-nanowire silicon solar cells,, Nano Lett. 8 (2008) 710-714. doi:10.1021/nl072622p.

[6] T. Stelzner, M. Pietsch, G. A. a, F. Falk, E. Ose, S. Christiansen, Silicon nanowire-based solar cells, Nat. Nanotechnol. 19 (2008) 295203. doi:10.1088/0957-4484/19/29/295203.

35 [7] L. Hu, G. Chen, Analysis of optical absorption in silicon nanowire arrays for photovoltaic applications, Nano Lett. 7 (2007) 3249-3252. doi:10.1021/nl071018b.

[8] J. Zhu, Z. Yu, G. Burkhard, C. Hsu, S. Connor, Y. Xu, Q. Wang, M. M. Gehee, S. Fan, Y. Cui, Optical absorption enhancement in amorphous silicon nanowire and nanocone arrays, Nano Lett. 9 (2009) 279-282. doi:10.1021/nl802886y.

[9] J. Li, H. Yu, S. Wong, G. Zhang, X. Sun, P. Lo, D. Kwong, Si nanopillar array optimization on si thin films for solar energy harvesting, Appl. Phys. Lett. 95 (2009) 033102. doi:10.1063/1.3186046.

[10] Y. Huang, S. Chattopadhyay, C. P. Y. Jen, T. Liu, Y. Hsu, C. Pan, H. Lo, C. Hsu, C. L. Y. Chang, K. Chen, L. Chen, Improved broadband and quasi-omnidirectional anti-reflection properties with biomimetic silicon nanostructures, Nat. Nanotechnol. 2 (2007) 770-774. doi:10.1038/nnano.2007.389.

350 [11] S. Han, G. Chen, Optical absorption enhancement in silicon nanohole arrays for solar photovoltaics, Nano Lett. 10 (2010) 1012-1015. doi: $10.1021 / \mathrm{n} 1904187 \mathrm{~m}$. 
[12] Y. Cui, J. Xu, K. Fung, Y. Jin, A. Kumar, S. He, X. Fang, Ultrabroadband light absorption by a sawtooth anisotropic metamaterial slab, Nano Lett. 12 (2012) 1443-1447. doi:10.1021/nl204118h.

[13] Q. Liang, T. Wang, Z. Lu, Q. Sun, Y. Fu, W. Yu, Metamaterialbased two dimensional plasmonic subwavelength structures offer the broadest waveband light harvesting, Adv. Opt. Mater. 1 (2013) 43-49. doi:10.1002/adom.201200009.

[14] J. Horng, C.-F. Chen, B. Geng, C. Girit, Y. Zhang, Z. Hao, H. A. Bechtel, M. Martin, A. Zettl, M. F. Crommie, Y. R. Shen, F. Wang, Drude conductivity of dirac fermions in graphene, Phys. Rev. B 83 (2011) 165113. doi:10.1103/PhysRevB.83.165113.

[15] Z. Q. Li, E. A. Henriksen, Z. Jiang, Z. Hao, M. C. Martin, H. L. S. P. Kim, D. N. Basov, Dirac charge dynamics in graphene by infrared spectroscopy, Nat. Phys. 4 (2008) 532. doi:10.1038/nphys989.

[16] L. Ju, B. Geng, J. Horng, C. Girit, M. Martin, Z. Hao, H. A. Bechtel, A. Z. X. Liang, Y. R. Shen, F. Wang, Graphene plasmonics for tunable terahertz metamaterials, Nat. Nanotechnol. 6 (2011) 630. doi:10.1038/NNANO.2011.146.

[17] F. H. L. Koppens, D. E. Chang, F. J. G. de Abajo, Graphene plasmonics: A platform for strong lightcmatter interactions, Nano Lett. 11 (2011) 3370. doi:10.1021/nl201771h.

[18] P. Tassin, T. Koschny, M. Kafesaki, C. M. Soukoulis, A comparison of graphene, superconductors and metals as conductors for metamaterials and plasmonics, Nat. Photonics 6 (2012) 259. doi:10.1038/NPHOTON.2012.27.

[19] S. Thongrattanasiri, F. H. L. Koppens, F. J. G. de Abajo, Complete optical absorption in periodically patterned graphene, Phys. Rev. Lett. 108 (2012) 047401. doi:10.1103/PhysRevLett.108.047401. 
[20] A. Y. Nikitin, F. Guinea, F. J. Garcia-Vidal, L. Martin-Moreno, Fields radiated by a nanoemitter in a graphene sheet, Phys. Rev. B 84 (2011) 195446. doi:10.1103/PhysRevB.84.195446.

[21] R. Filter, M. Farhat, M. Steglich, R. Alaee, C. Rockstuhl, F. Lederer, Tunable graphene antennas for selective enhancement of thz-emission, Opt. Express 21 (2013) 3737. doi:10.1364/0E.21.003737.

[22] B. Vasić, G. Isić, , R. Gajić, Localized surface plasmon resonances in graphene ribbon arrays for sensing of dielectric environment at infrared frequencies, J. Appl. Phys. 113 (2013) 013110. doi:10.1063/1.4773474.

[23] L. Britnell, R. Gorbacev, R. Jalil, B. Belle, F. Schedin, A. Michenko, T. Georgiou, M. Katsnelson, L. Eaves, S. Morsov, N. Peres, J. Leist, A. geim, K. Novoselov, L. Ponomarenko, Field-effect tunneling transistor based on vertical graphene heterostructures, Science 335 (2012) 947. doi:10.1126/science.1218461

[24] M. Liu, X. Yin, X. Zhang, Double-layer graphene optical modulator, Nano Lett. 12 (2012) 1482. doi:10.1021/nl204202k.

[25] H. Yan, X. Li, B. Chandra, G. Tulevski, Y. Wu, M. Freitag, W. Zhu, P. Avouris, F. Xia, Tunable infrared plasmonic devices using graphene/insulator stacks, Nat. Nanotechnol. 7 (2012) 330. doi:10.1038/NNANO.2012.59.

[26] I. Iorsh, I. Mukhin, I. Shadrivov, P. Belov, Y. Kivshar, Hyperbolic metamaterials based on multilayer graphene structures, Phys. Rev. B 87 (2013) 075416. doi:10.1103/PhysRevB.87.075416

[27] T. Stauber, G. Gómez-Santos, Plasmons and near-field amplification in double-layer graphene, Phys. Rev. B $85 \quad(2012) 075410$. 
[28] G. G. de la Cruz, Role of metallic substrate on the plasmon modes in double-layer graphene structures, Solid. State Commun. 213 (2015) 6-9. doi:10.1103/PhysRevB.85.075410.

[29] A. Benz, S. Campaine, M. Moseley, J. Wierer, A. Allerman, J. Wendt,

[36] Q. Hao, Y. Zeng, X. Wang, Y. Zhao, B. Wang, I. Chiang, D. H. Werner, V. Crespi, T. J. Huang, Effects of intrinsic fano interference on surface 
enhanced raman spectroscopy: Comparison between platinum and gold, Appl. Phys. Lett. 97 (2010) 193101. doi:10.1021/jp105276w.

[37] M. J. Dodge, Refractive properties of magnesium fluoride, Appl. Opt. 23 (1984) 1980-1985. doi:10.1364/A0.23.001980.

[38] P. Berini, Plasmon polariton modes guided by a metal film of finite width, Opt. Lett. 24 (1999) 1011-1013. doi:10.1364/0L.24.001011.

440 [39] G. W. Hanson, Dyadic greens functions and guided surface waves for a surface conductivity model of graphene, J. Appl. Phys. 103 (2008) 064302. doi:10.1063/1.2891452.

[40] M. Amin, M. Farhat, H. Bă̆ci, A dynamically reconfigurable fano metamaterial through graphene tuning for switching and sensing applications, Sci. Rep. 3 (2013) 2105. doi:10.1038/srep02105.

[41] K. Yang, S. Liu, S. Arezoomandan, A. Nahata, B. S. Rodrigueza, Graphene-based tunable metamaterial terahertz filters, Appl. Phys. Lett. 105 (2014) 093105. doi:10.1063/1.4894807.

[42] A. G. Ardakani, Tunability of absorption with temperature in the terahertz regime based on photonic crystals containing graphene and defect insb layers, Eur. Phys. J. B 88 (2015) 166. doi:10.1140/epjb/e2015-60233-0.

[43] Y. Ekinci, A. Christ, M. Agio, O. Martin, H. Solak, J. L Electric and magnetic resonances in arrays of coupled gold nanoparticle in-tandem pairs, Optics Express 16(17) (2008) 13287-13295. doi:10.1364/0E.16.013287.

455 [44] C. Casiraghi, A. Hartschuh, E. Lidorikis, H. Qian, H. Harutyunyan, T. Gokus, K. S. Novoselov, A. C. Ferrari, Rayleigh imaging of graphene and graphene layers, Nana Lett. 7 (2007) 2711-2717. doi:10.1021/n1071168m.

[45] R. R. Nair, P. Blake, A. N. Grigorenko, K. S. Novoselov, T. J. Booth, T. Stauber, N. M. R. Peres, A. K. Geim, Fine structure constant defines visual transparency of graphene, Since 320(5881) (2008) 1308-1308. doi:10.1126/science.1156965 
[46] A.K.Geim, K.S.Novoselove, The rise of graphene, Nature 6 (2007) 183-191. doi:10.1038/nmat1849.

[47] N. Emani, T. Chung, X. Ni, V. A. Kildishev, P. Chen, A. Boltasseva, Electrically tunable damping of plasmonic resonances with graphene, Nano Lett. 12 (2012) 5202-5206. doi:10.1021/nl302322t.

[48] Y. Zhao, X. Li, Y. Du, G. Chen, Y. Qu, J. Jiang, Y. Zhu, Strong lightmatter interactions in sub-nanometer gaps dened by monolayer graphene: toward highly sensitive sers substrates, Nanoscale 6 (2014) 11112-11120. doi:10.1039/c4nr03152k

[49] G. Konstantatos, M. Badioli, L. Gaudreau, J. Osmond, M. Bernechea, F. P. G. de Arquer, F. Gatti, F. Koppens, Hybrid graphene-quantum dot phototransistors with ultrahigh gain, Nat. Nanotechnol. 7 (2012) 363-368. doi:10.1038/NNANO.2012.60.

475 [50] Z. Fang, Z. Liu, Y. Wang, Plasmon-induced doping of graphene, Nano Lett. 12 (2012) 3808-3813. doi:10.1021/nn304028b.

[51] J. Reed, H. Zhu, A. Zhu, C. Li, E. Cubukcu, Grapheneenabled silver nanoantenna sensors, Nano Lett. 12 (2012) 4090-4094. doi:10.1021/n1301555t.

${ }_{480}[52] \mathrm{W} . \mathrm{Xu}, \mathrm{N} . \mathrm{Mao}, \mathrm{J}$. Zhang, Graphene: A platform for surfaceenhanced raman spectroscopy, Small 9 (2013) 1206-1224. doi:10.1002/smll.201203097.

[53] W. Zhou, K. Li, C. Song, P. Hao, M. Chi, M. Yu, , Y. Wu, Polarizationindependent and omnidirectional nearly perfect absorber with ultra-thin 2d subwavelength metal grating in the visible region, Opt. Express 23(11) (2015) A413-A418. doi:10.1364/OE.23.00A413.

[54] X. Li, L. Y. Yang, C. G. Hu, X. G. Luo, M. H. Hong, Tunable bandwidth of band-stop filter by metamaterial cell coupling in optical frequency, Opt. Express 19(6) (2011) 5283-5289. doi:10.1364/OE.19.005283 
490 [55] M. N. Abbas, C. W. Cheng, Y. C. Chang, M. H. Shih, An omni-directional mid-infrared tunable plasmonic polarization filter, Nanotechnology 23(44) (2012) 444007. doi:10.1088/0957-4484/23/44/444007. 